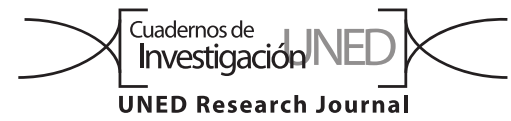

\title{
Estructura de hato y manejo nutricional, sanitario, reproductivo y ambiental del caballo iberoamericano en Costa Rica
}

\author{
Grethel Solano Mora' ${ }^{2}$ Rodolfo WingChing-Jones ${ }^{2}$ \\ 1. Criadero Los Tres Potrillos S.A.; gsolano17@outlook.com \\ 2. Centro de Investigación en Nutrición Animal (CINA), Escuela de Zootecnia, Universidad de Costa Rica; rodolfo.wingching@ucr.ac.cr
}

Recibido 03-IV-2018 • Corregido 05-VII-2018 • Aceptado 06-VIII-2018

\begin{abstract}
Herd structure, and nutritional, sanitary, reproductive and environmental management of Iberoamerican horses in Costa Rica. Knowing the nutritional, sanitary, reproductive and facilities management of animals of zootechnical interest, is the basis for improving the systems. In October 2016 and April 2017 we carried out a diagnosis of 16 Ibero-American horse breeding farms located in the provinces of Alajuela, Cartago, San José and Heredia in Costa Rica. We found a female: male ratio of 1,7 ; and seven crosses, the most frequent was between a female Costa Rican Saddle horse and a male purebred Spanish horse. There were nine riding arena sizes (six for the stables, ten for the washing facilities and ten forages). A positive balance of protein and energy in the diets could increase body condition. Natural mating, artificial insemination, embryo transfer, and heat detection and synchronization, are all implemented. Deworming and vaccination, and preventive management of bedding, are recorded by the farm workers (62\% are trained on horse care and management). These horse breeders have developed valid knowledge through self-training, but there is room for improvement in the diagnosis of parasitic diseases, veterinary recommendations and feeding practices.
\end{abstract}

Keywords: Facility, crosses, handling, reproduction, feeding.
RESUMEN: Conocer el manejo nutricional, sanitario, reproductivo y de las instalaciones de los animales de interés zootécnico es la base para mejorar los sistemas. En octubre de 2016 y abril de 2017 llevamos a cabo el diagnóstico de 16 granjas hípicas iberoamericanas ubicadas en las provincias de Alajuela, Cartago, San José y Heredia de Costa Rica. Encontramos una relación hembra: macho de 1,7; y siete cruces, el más frecuente fue entre una hembra costarricense de paso y un macho de la raza pura española. Hubo nueve tamaños para el picadero (seis para las cuadras, diez para los bañaderos y diez forrajes). Un balance positivo de proteína y energía en las dietas podría aumentar la condición corporal. Se implementan la monta natural, inseminación artificial, transferencia de embriones y la detección y sincronización de celos. La desparasitación y la vacunación, y el manejo preventivo de la cama, son registrados por los trabajadores del campo (el 62\% están capacitados en el cuidado y manejo del caballo). Estos criadores de caballos han desarrollado conocimientos válidos a través de la autoformación, pero hay margen de mejora en el diagnóstico de enfermedades parasitarias, recomendaciones veterinarias y prácticas de alimentación.

Palabras clave: Instalaciones, cruces, manejo, reproducción, alimentación.
La historia del caballo en Costa Rica se remonta a la época de la conquista española, momento donde los españoles trajeron sus caballos para transporte y carga. Con el pasar de los años la cría equina se desarrolló en la zona de Cartago y en el Valle Central por las necesidades que se tenían en labores cotidianas como el transporte y actividades agropecuarias (Carazo, 1994). En el año 1880, el gobierno dictó leyes que permitieron la importación de ganado, dándose así la importación de caballos de distintas razas (Seravalli, 2011).

En el año 2014 en Costa Rica, según el censo agropecuario existían 66942 equinos, distribuidos en Guanacaste $(28,24 \%)$, Alajuela $(23,29 \%)$, Puntarenas (20,85\%) y San José $(9,70 \%)$ (INEC, 2015). En Costa Rica existen diferentes razas de caballos entre las que se encuentran la costarricense de paso, pura raza Española, Cuarto de Milla, Appaloosa, Pinto americano, Peruano, Paso Fino colombiano, Árabe e Iberoamericano entre otras (Carazo, 1994).

Para inicios del Siglo XX Ilegaron al país equinos importados de las razas Peruana y pura raza Española, animales que dan origen a lo que hoy en día se conoce como el caballo costarricense de paso. Este caballo se cruzaría con caballos ibéricos como el pura raza Española y el Lusitano, cruce que generaría el caballo Iberoamericano (Seravalli, 2011). Actualmente esta raza cuenta con 4638 animales inscritos en la Asociación Centroamericana de Criadores de Caballos de Raza Iberoamericana (ASOIBERO) (Peña, 
2016 , comm pers). Dicha asociación es la encargada de llevar el registro genealógico de estos animales como lo establece el Gobierno de Costa Rica por medio del Ministerio de Agricultura y Ganadería en los decretos №19400-MAG del año 1989, № 23685-MAG del año 1994 y el № 20972-MAG del año 1991. En el país, animales iberoamericanos están siendo exportados a países como México, Guatemala y Nicaragua generando ingresos superiores a los $\$ 4$ millones al año (López, 2008).

El desconocimiento de las actividades técnicas que se desarrollan en esta actividad creciente, como el manejo nutricional, requerimientos nutricionales, consumo de materia seca, protocolos sanitarios, tipos de instalaciones, entre otras acciones, genera la necesidad de diagnosticar esta actividad para el desarrollo de un conocimiento básico, que sirva para el desarrollo de herramientas que ayuden al manejo zootécnico de estos animales en sus distintas etapas fisiológicas y áreas de trabajo. Es por esta razón que generar el conocimiento de la estructura de hato, las instalaciones, el manejo nutricional, sanitario y de los animales permite desarrollar diferentes prácticas para la producción eficiente y rentable de caballos de raza Iberoamericana.

Por tal motivo, el objetivo del presente trabajo fue diagnosticar 16 sistemas de producción de caballo iberoamericano en Costa Rica con el fin de conocer cómo se manejan estos animales, para así generar un conocimiento base para futuras investigaciones sobre el tema.

\section{MÉTODOLOGÍA}

Recolección de datos.: El estudio se realizó entre septiembre de 2016 y marzo de 2017. Se encuestó a un total de 16 criaderos asociados a ASOIBERO, ubicados en diferentes zonas del país. Los datos se recolectaron por medio de un instrumento tipo encuesta compuesta por 65 preguntas sobre la estructura del hato, manejo de los animales, manejo sanitario, reproducción, instalaciones, mano de obra y manejo nutricional (Fig. 1). La encuesta fue realizada en conjunto con el dueño de los animales como con el encargado del manejo, con la intención de unificar criterios.

\section{RESULTADOS}

Ubicación y años de operación: La mayoría de los criaderos (62\%) se ubica en la provincia de Alajuela, sin embargo, también hay criaderos en Heredia, San José y Cartago. Dichos criaderos tienen entre 7 y 46 años de operar con un promedio de $21 \pm 13$ años de operación, donde el 19\%(3) presenta menos de 10 años de funcionamiento, el 37\%(6) de los criaderos presentan entre 10 y 20 de funcionamiento; entre 20 y 30 años de operar se encuentra el $25 \%$ (4) de los entrevistados y el $19 \%$ (3) de los sistemas visitados, registran más de 40 años de funcionamiento.

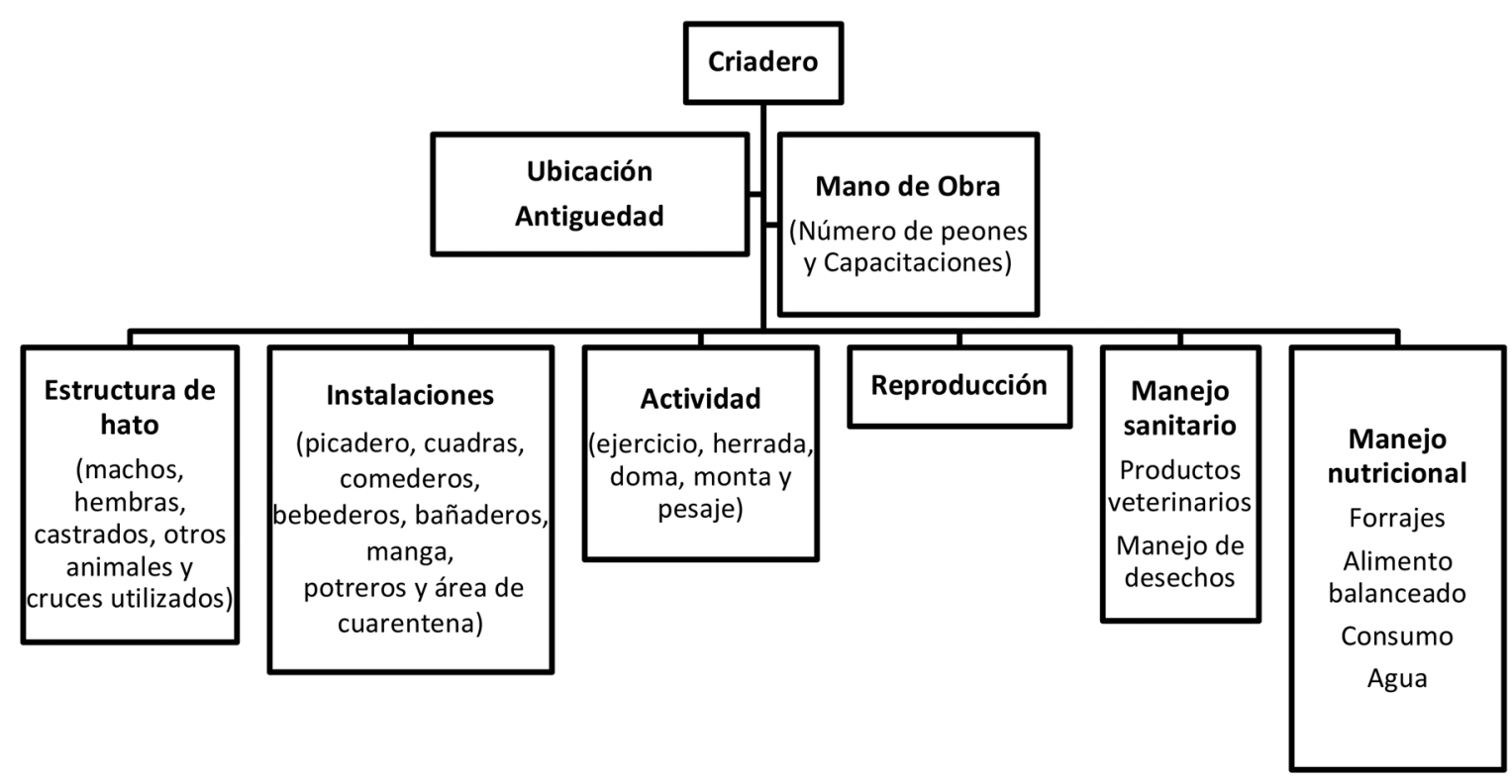

Fig. 1. Componentes del instrumento tipo encuesta aplicado a los propietarios de los criaderos de caballo Iberoamericano visitados. 
CUADRO 1

Distribución promedio del hato de caballos en los criaderos consultados

\begin{tabular}{|c|c|c|c|}
\hline Animal & Cantidad & Mínimo & Máximo \\
\hline Hembras & $11 \pm 10$ & 0 & 40 \\
\hline Machos & $6 \pm 5$ & 1 & 20 \\
\hline Castrados & $1 \pm 1$ & 0 & 4 \\
\hline Total de caballos Iberoamericanos & $18 \pm 15$ & 2 & 64 \\
\hline Total de caballos (incluye otras razas) & $29 \pm 22$ & 6 & 84 \\
\hline
\end{tabular}

Hato de animales: En el Cuadro 1, se observa la distribución promedio de los animales dentro de los criaderos según su sexo y raza. Lo que permite obtener una relación hembra: macho de 1,7. Además, se resalta, que en promedio cada criadero visitado presenta 11 animales que no son de la raza iberoamericana.

La mayoría de criadores optan por utilizar hembras de la raza Costarricense de Paso con machos de la Pura Raza Española para la obtención de caballos Iberoamericanos, sin embargo, también se utilizan otros cruces para la obtención de los mismos (Fig. 2).

Instalaciones: Las instalaciones de los diferentes criaderos están conformadas por el picadero, las cuadras, potreros, área de cuarentena y los bañaderos y mangas. El ochenta y siete por ciento de los criaderos señala tener picadero en sus fincas y de estos el $21 \%$ señalan que el mismo es techado. Del total de criaderos encuestados, el $36 \%$ señala que no conoce la profundidad del piso del picadero y el $72 \%$ señala que utilizan arena para el mismo. Otros materiales utilizados son piedra (14\%), quintilla (7\%) y tierra (7\%). En el caso de las dimensiones del picadero, se registraron 9 diseños diferentes, los cuales ofrecen entre 150 hasta $1250 \mathrm{~m}^{2}$, donde la más utilizada, es la que genera $800 \mathrm{~m}^{2}$, con $20 \mathrm{~m}$ de largo por 40 de ancho.

El $100 \%$ de los criadores señala que posee cuadras en sus instalaciones y es el lugar donde mantienen a los animales. Mientras que el $80 \%$ de los mismos señalan que las cuadras presentan dimensiones mayores a los $9 \mathrm{~m}^{2}$. En el caso de la presencia de un área para la cuarentena de animales nuevos que ingresen al sistema, el 56\% señaló que no poseen este espacio dentro de sus instalaciones. En cuanto a la cama utilizada para las cuadras el $75 \%$ de los criadores utilizan aserrín y viruta. Sin embargo, el 31\% de los criadores señala que no conoce la profundidad de la misma, la cual fluctuó entre 3 a $25 \mathrm{~cm}$ (69\% restante). En el caso de los comederos y las heneras, el $44 \%$ y $56 \%$ respectivamente, señalan que lo colocan a nivel del suelo, mientras que en el caso de los bebederos el $50 \%$ de los criaderos señala que lo colocan a 1 metro de altura.

En el caso de los bañaderos, es un área presente en todos los sistemas para el baño de los animales, de igual manera, es utilizada como manga de trabajo. Se

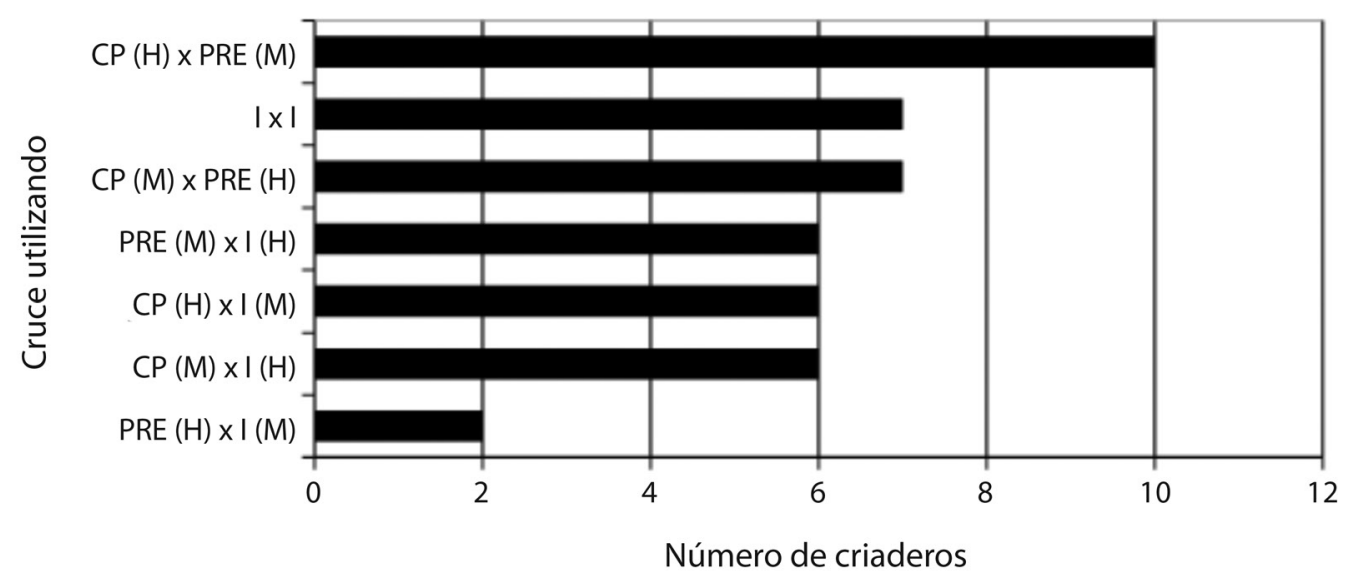

Fig. 2. Cruces utilizados en los criaderos asociados a ASOIBERO para la obtención de caballo de raza lberoamericana. $\mathrm{CP}=$ Costarricense de Paso, PRE= Pura Raza Española, I= Iberoamericano, H= Hembra, M= Macho. 
determinaron seis medidas diferentes para el ancho y siete para el largo, con un área de trabajo que fluctuó entre 1,36 a $16 \mathrm{~m}^{2}$.

Por último, el $100 \%$ de los sistemas poseen potreros en sus fincas para el pastoreo de sus animales, sin embargo, el 31\% señala que no conocen el área destinada a la producción de forraje. Estas áreas fluctúan entre una hectárea hasta 10ha, según las dimensiones del criadero y el número de animales presentes. En el caso del cultivo, que se maneja en estos sistemas, se registran 9 forrajes, donde el de mayor frecuencia fue el pasto Estrella africana $(47,6 \%)$, seguido por los forrajes Braquipara (14,3\%), Ratana (9,5\%), Jaragua (4,7\%), Swazi $(4,7 \%)$, Limpograss $(4,7 \%)$, Massai $(4,7 \%)$, Toledo (4,7\%) y Mombaza $(4,7 \%)$.

Manejo de los animales: El 50\% de los criaderos señalan que sus animales se entrenan tres veces por semana, con un promedio de $35 \pm 15 \mathrm{~min}$ con un rango de 20 a $60 \mathrm{~min}$. En el caso del baño, el $44 \%$ de los criadores lo realizan tres veces por semana, inclusive se encontró un sistema de producción que baña a los animales cuando sea necesario y dos sistemas que los bañan todos los días. Mientras el herraje, se realiza en el 50\% de los criadores cada dos meses (ocho semanas).

La doma de estos animales se da en dos etapas, la doma a diestro y la doma montada. En el caso de la doma a diestro, los animales inician a la edad de $8 \pm 9,5$ meses con un intervalo de 0,7 a 36 meses. El $44 \%$ de los criadores señalan que inician la doma a los 6 meses. En el caso de la doma montada, los animales inician a los $3 \pm 0,13$ años con un intervalo de los 3 a los 3,5 años.

El $67 \%$ de los criadores señala que realizan la práctica de impronta (imprinting) a la hora de nacimiento de los potros. En el caso del pesaje de los animales en los criaderos, el $56 \%$ de los criadores señalo que si realiza esta práctica y el $50 \%$ lo realiza de forma semestral. Siendo la cinta de predicción de peso el método más utilizado con el $37 \%$, seguido por la romana (25\%) y ecuaciones predictoras $(25 \%)$ y por último el método visual con el $13 \%$ de los criaderos consultados.

Reproducción: Todos los criaderos consultados señalan que utilizan la monta natural para la reproducción de sus animales, sin embargo, también señalaron que utilizan prácticas como inseminación artificial $(31,2 \%)$, transferencia de embriones $(6,2 \%)$, detección de celos $(50 \%)$ y sincronización de celos (50\%). Estas prácticas se realizan tanto con animales propios (o') como prestados de otros sistemas de producción.
Para la edad al primer servicio de las yeguas se deter-

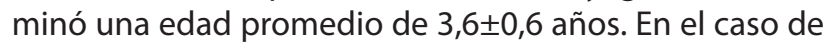
la edad al destete, los potros en los criaderos de caballos de raza Iberoamericana, son destetados a los $6 \pm 1$ meses.

Manejo sanitario: El 100\% de los criadores vacunan a los potros recién nacidos con la vacuna antitetánica, mientras que solo uno, vacuna contra la encefalitis. La presencia de un botiquín de primeros auxilios, se determinó en el $94 \%$ de los criaderos. Dentro de los grupos de productos, se encontraron analgésicos y antiinflamatorios (inyectables y tópicos), antibióticos, vitaminas, suplementos orgánicos, desparasitantes, desinfectantes, vacunas antitetánicas, cicatrizantes, garrapaticidas, larvicidas, alcohol y suero. En el caso de las desparasitaciones, el 50\% de los criadores lo realizan cada 3 meses de manera rutinaria, ya que el $100 \%$ de los mismos señalaron que no realizan análisis de heces. Los criadores señalaron que rotan los productos ( $100 \%$ de los criaderos) y que utilizan los siguientes ingredientes activos durante las desparasitaciones, ivermectina, doramectina, febendazol, albendazol y praziquantel.

La limpieza de las camas es otra medida preventiva que se realizan en estos sistemas de producción, donde el $50 \%$ la realiza una vez a la semana, el $25 \%$ de los sistemas la realizan entre dos y tres días, y el restante $50 \%$ la realiza de forma diaria. En todos los casos, el material de la cama, es procesado de forma natural mediante la técnica del compostaje, posterior a la culminación de su proceso, momento en el cual, el material no presenta calentamiento, es utilizado como enmienda en las áreas dedicadas al cultivo del forraje.

Manejo nutricional: Los criadores de caballos Iberoamericanos señalan que utilizan los siguientes forrajes para la alimentación de sus animales: Mombaza (Panicum maximum cv. Mombaza), Massai (Panicum maximum Cv. Massai), Swazi (Digitaria swazilandensis), Brachipará (Brachiaria arrecta x Brachiaria mutica), Transvala (Digitaria decumbens Stent. Cv. Transvala) y Estrella africana (Cynodon nlemfuensis). El 75\% por ciento de los criadores señalan que utilizan estos forrajes en fresco y el $69 \%$ de estos señala que utiliza también heno de Transvala, ya sea como fuente única de forraje o como complemento. En promedio se ofrecen $9,33 \pm 4,5 \mathrm{~kg}$ de heno al día con un rango de 3 a $18 \mathrm{~kg}$ y $33,7 \mathrm{~kg}$ en promedio de forraje verde.

La totalidad de los criadores ofrecen a sus animales alimento balanceado, suministran entre 1 y $4,5 \mathrm{~kg}$ de alimento por día con un promedio de $3,4 \pm 1 \mathrm{~kg}$, el $53 \%$ de los criadores prefieren este alimento peletizado, el 
CUADRO 2

Cálculo del balance nutricional de un caballo Iberoamericano adulto con un peso de 446,23kg y ejercicio moderado, consumiendo heno, alimento balanceado y pastoreando Estrella africana

\begin{tabular}{|c|c|c|c|c|}
\hline & & $\begin{array}{l}\text { Materia Seca } \\
(\%)\end{array}$ & $\begin{array}{l}\text { Proteína Cruda } \\
\text { (PC \%) }\end{array}$ & $\begin{array}{l}\text { Energía Digestible } \\
\text { (ED Mcal } / \mathrm{kg})\end{array}$ \\
\hline \multirow{3}{*}{$\begin{array}{l}\text { Composición nutricional de los } \\
\text { componentes de la dieta }\end{array}$} & Estrella africana & 23,5 & 13,33 & 2,37 \\
\hline & Heno Transvala & 80 & 3,42 & 2,05 \\
\hline & Alimento balanceado & 87 & 14 & 3,2 \\
\hline \multirow{4}{*}{$\begin{array}{l}\text { Requerimientos nutricionales } \\
\text { del caballo }\end{array}$} & & & PC (g/día) & ED (Mcal/día) \\
\hline & Mantenimiento* & & 562,25 & 14,79 \\
\hline & Ejercicio moderado & & 78,98 & 6,02 \\
\hline & Total & & 641,23 & 20,80 \\
\hline \multirow[t]{6}{*}{ Balance nutricional } & & & PC g/día & ED Mcal/día \\
\hline & Aporte Alimento Balanceado & & 487,2 & 11,14 \\
\hline & Aporte Estrella africana & & 1053,29 & 18,77 \\
\hline & Aporte Heno Transvala & & 246,24 & 14,76 \\
\hline & Total de Aportes & & 1786,73 & 44,67 \\
\hline & Balance & & $+1145,5$ & $+23,87$ \\
\hline
\end{tabular}

*NRC. Nutrient requeriments of horses (2017).

$41 \%$ extrusado y el $6 \%$ en harina. Las concentraciones de nutrimentos de estos alimentos fluctúan entre 12 y $14 \%$ de proteína cruda, de 3 a $4 \%$ de extracto etéreo, de 6 a15\% de fibra cruda, entre 3.000 y $3.200 \mathrm{kcal} / \mathrm{kg}$ de energía digestible, 0,3 a 1,3\% de calcio, 0,4 a $0,46 \%$ de fósforo y entre 0,2 a $1,5 \%$ de sal $(\mathrm{NaCl})$. Según las cantidades suministradas promedio y usando de referencia un animal de $446,23 \mathrm{~kg}$ de peso (peso promedio de un caballo Iberoamericano adulto), se obtiene que los animales podrían ganar condición corporal, debido a que presentan un balance positivo en el consumo de proteína cruda y de energía digestible (Cuadro 2).

El 63\% de los criadores alimenta a sus animales 2 veces al día. El $100 \%$ de los mismos brindan agua a libre consumo, mientras que solo el $25 \%$ realizan análisis de agua para verificar la calidad de la misma. El responsable de determinar la dieta de los caballos en los distintos criaderos es el mismo criador, situación que se refleja en el $63 \%$ de las veces, seguido por el veterinario con el $17 \%$, el vendedor de alimento con el $12 \%$ y el zootecnista con el $6 \%$.

Mano de obra: Los criadores encuestados contratan entre 1 y 4 peones con un promedio de 2 personas, que presenten experiencia en el cuido y manejo de los animales. De los 16 criaderos visitados, el $62 \%$ de estos trabajadores están capacitados, situación que en el $87 \%$ de los dueños de los criadores señala indispensable, para el éxito de la actividad y el bienestar de los animales. Para el trabajo y adiestramiento de los animales, el $94 \%$ de los criaderos cuentan con montador o entrenador para sus caballos. Además, se estimó que se requiere un peón por cada 12 equinos. En el caso de la visita del especialista en medicina veterinaria, la mayoría de los criaderos determina que las visitas son cuando se presente un evento especial, pero, de forma rutinaria las visitas se dan desde una vez a la semana hasta cada seis meses, según lo considere el propietario del sistema.

\section{DISCUSIÓN}

El crecimiento en el registro y número de sistemas dedicados a la producción del caballo Iberoamericano, se podría relacionar a que la asociación ASOIBERO cuenta con 26 años de operación al año 2017, lo que muestra que desde su fundación se impulsó el desarrollo de esta raza en el país, ya que la mayoría de criadores inician operaciones posteriores a esta fecha. Mientras que los criaderos que presentan mayor antigüedad en el país, el registro de animales Iberoamericanos estaba relacionado al tipo de caballo con el cual iniciaron la actividad de crianza de caballos debido a que en los casos del caballo pura raza española y costarricense de paso podían ser registrados, desde el año 1975 y 1972 respectivamente (ASOCPRE, 2017; DETOPE, 2017).

Debido al proceso de obtención del animal Iberoamericano, los criadores de caballo iberoamericano tienen en sus ganaderías animales de otras razas con el fin de realizar los cruces respectivos. La mayoría de criadores optan por utilizar hembras de la raza costarricense 
de paso con machos de la raza pura española, debido a que el caballo de pura raza española macho debe tener según su patrón racial un mínimo de 60 pulgadas a la altura a la cruz y las hembras de raza costarricense de paso deben tener un temperamento brioso y noble, características deseadas en el patrón racial de los caballos Iberoamericano (ANCCE, 2017; ASCACOPA, 2015).

Al analizar las instalaciones, determinadas en esta investigación, el tipo de piso, y las dimensiones de las cuadras $\left(9 \mathrm{~m}^{2}\right)$, concuerdan con lo descrito por Acero (2016) y León (2007) respectivamente. De igual manera, en el uso del aserrín y viruta, por ser materiales absorbentes y por su costo económico (Sáenz, 2008; León, 2007; Acero, 2016). Siendo el menos recomendado el aserrín por ser más polvoso, y esto puede ocasionar alergias en los caballos.

Según Holst y Bolze (2010), es recomendable brindar el alimento y forraje a nivel del suelo, como se observó en la presente investigación, lo cual lo relacionan con las afirmaciones de Loving (2010), donde cuestiona que la acción de colocar los comederos a una mayor altura, debido a que existe la posibilidad de provocar problemas respiratorios y cólicos por ser una posición antinatural para alimentarse.

Los caballos de competencia de raza Iberoamericana requieren estar preparados para el trabajo al trote por periodos de tiempo que van de 10 a 40 minutos (ASOIBERO, 2017). Zamora (2013) señala que es recomendado trabajar los animales de 2 a 3 veces por semana durante 30 minutos, lo que concuerda con los resultados de esta investigación. El intervalo de herraje utilizado por los criadores de caballo Iberoamericano en Costa Rica concuerda con lo señalado por Acero (2016), Sáenz (2008) y León (2007) quienes indican que se debe realizar entre un rango de 4 y 8 semanas. En el caso de la doma de los animales montados, los periodos recopilados en esta investigación concuerdan con lo señalado por Acero (2016) y León (2007), quienes indican que este proceso se debe realizar a los 3 años. En el caso de realizar la impronta, la cual es una acción basada en estímulos mediante el tacto del humano y la cría en las primeras dos horas de vida del animal, para que asocie estos contactos como situaciones positivas, esta práctica se realiza en 10 de los criaderos visitados, lo que podría facilitar procesos posteriores como el herraje y la doma en estos sistemas (Acero, 2016; Cano, 2010; León, 2007),

La edad de destete, determinada en esta investigación concuerda con la edad recomendada para este proceso por León (2007) y Acero (2016). En cuanto a la edad al primer servicio, Sáenz (2008) recomienda que esta sea a los 2,5 años, edad menor a la determinada en el presente trabajo, esto debido a que los criadores deseen que las yeguas tengan un mejor desarrollo y no sea al mismo tiempo que el inicio de la doma montada.

La vacunación al momento del nacimiento contra el tétano es una práctica acertada por los criadores de caballo Iberoamericano, ya que esta enfermedad es causada por una bacteria común en el entorno de los caballos (Hayes, 2009). Por otro lado, en el programa de desparasitaciones, solo 5 de los 16 criaderos, realizan exámenes de heces para programar la aplicación de desparasitantes, en este sentido, Hayes (2008) señala que se deben realizar análisis de heces para conocer el momento óptimo de la desparasitación con el fin de evitar resistencia en los parásitos, por lo cual sería una práctica a implementar en los criaderos consultados.

En el caso de la determinación de la dieta ofrecida a los animales en los criaderos visitados, en la mayoría de los casos (11), la proporción de los insumos alimenticios es determinada por el dueño del criadero, y en una menor proporción por el médico veterinario que visita el sistema (3), el vendedor de alimento balanceado (1) y el zootecnista (1), situación que provoca que los requerimientos de proteína cruda y energía de los animales sean superados, con el agravante de que los animales podrían presentar un sobrepeso, y ser propensos a lesiones. El apoyo en un nutricionista o zootecnista, es una posibilidad de mejora, para satisfacer los requerimientos de los animales, según su peso y a la actividad física a la cual es sometido.

Con la presente investigación se permitió realizar un diagnóstico del estado actual y de las prácticas que realizan los criaderos de caballos Iberoamericano en Costa Rica. Se concluye que los criadores de caballo Iberoamericano tienen conocimientos propios por medio de auto capacitación, ya que realizan varias prácticas de manera correcta, sin embargo, todavía existen otras que se pueden mejorar como lo es la implementación del análisis de heces para determinar el tipo de desparasitante, la frecuencia y métodos de pesaje, y la utilización de un profesional en nutrición animal entre otras.

\section{AGRADECIMIENTOS}

Agradecemos a la ASOIBERO, sus criadores asociados y los montadores y jaladores de los animales, los cuales brindaron todas las facilidades posibles para poder realizar el presente trabajo. 


\section{REFERENCIAS}

Acero, P. (2016). Planificación y Manejo de la Explotación Equina. Consejería de Agricultura y Ganadería: España.

ANCCE (Asociación Nacional de Criadores de Caballos de Pura Raza Española). (2017). Patrón Racial de la Raza Pura Raza Española. Asociación Nacional de Criadores de Caballos de Pura Raza Española (ANCCE), Sevilla, España. Recuperado de www.ancce.es

ASCACOPA (Asociación de criadores del caballo costarricense de Paso). (2015). Patrón Racial de la Raza Costarricense de Paso. Asociación de criadores del caballo costarricense de Paso (ASCACOPA), San José, Costa Rica. Recuperado de www.ascacopa.com

ASOCPRE (Asociación Costarricense de Criadores de Caballos de Pura Raza Española.) (2017). Historia de la Asociación Costarricense de Criadores de Caballos de Pura Raza Española. Asociación Costarricense de Criadores de Caballos de Pura Raza Española (ASOCPRE), San José Costa Rica. Recuperado de www.precostarica.org

ASOIBERO (Asociación Centroamericana de Criadores del Caballo Iberoamericano). (2017). Reglamento de juzgamiento del caballo de raza iberoamericano. San José, Costa Rica: ASOIBERO.

Cano, L. (2010). Cuidados del neonato equino. Mundo Hípico, $17,54-56$.

Carazo, M. (1994). Equinos. In Cortés, G (ed.). Atlas Agropecuario de Costa Rica (pp: 479-490). San José, Costa Rica: EUNED.
DETOPE. (2017). Historia de Asociación Costarricense de Criadores de Caballo Costarricense de Paso. DETOPE, San José, Costa Rica. Recuperado de www.detope.com

Hayes, K. (2009). Primeros auxilios y cuidados del caballo. Barcelona, España: Editorial Hispano Europea S.A.

Holst, A., \& Bolze, D. (2010). El cólico. Barcelona, España: Editorial Hispano Europea.

INEC (instituto Nacional de Estadísticas y Censo). (2015). VI Censo Nacional Agropecuario. San José, Costa Rica: INEC.

León, P. (2007). Manejo y Cuidado del Caballo. España: Escuela Universitaria de Ingeniería Técnica Agrícola INEA.

Lopéz, T. (2008). Los caballos ticos se van volando. El Financiero, San José Costa Rica. Recuperado de www.elfinancierocr. com

Loving, N. (2010). Todos los sistemas del caballo. Barcelona, España: Editorial Hispano Europea.

NRC. (2007). Nutrient requirements of horses. Washington, Estados Unidos: The National Academies press.

Sáenz, A. (2008). Zootecnia equina. Nicaragua: Universidad Nacional Agraria de Nicaragua.

Seravalli, G. (2011). Breve reseña histórica de la Asociación Centroamericana de Criadores de Caballo de Raza Iberoamericana (ASOIBERO). Mundo Hípico, 19(1), 50.

Zamora, A. (2013). El exceso de ejercicio y sus consecuencias. Mundo Hípico, 22, 46-48. 